\title{
A NUMERICAL STUDY OF BLOOD FLOW PATTERNS IN CEREBRAL ANEURYSMS AND THE CAUSAL RELATIONSHIP WITH PLATELET AGGREGATION
}

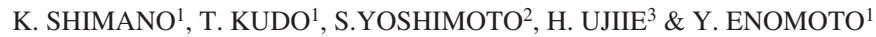 \\ ${ }^{1}$ Department of Mechanical Systems Engineering, Tokyo City University, Japan. \\ ${ }^{2}$ Tokyo University of Science, Japan. \\ ${ }^{3}$ Tokyo Rosai Hospital, Japan.
}

\begin{abstract}
Past statistical studies demonstrating the likelihood of slow blood flow in most ruptured aneurysms have suggested that thrombogenesis plays an important role in ruptures of cerebral artery aneurysms. In the authors' previous study, it was reported that the degree of platelet aggregation in an aneurysm had a significant correlation with the flow pattern in the aneurysmal dome. It is, therefore, crucial to investigate flow structures in various different aneurysms in order to understand better the relationship between thrombogenesis and ruptures. In this study, patterns of blood flow in three models of cerebral artery bifurcation aneurysms were numerically analysed and compared to discern the likelihood of platelet aggregation. The three model aneurysms had comparable aspect ratios (depth/neck width) but one model was larger in volume than the other two. Experimentally captured images of visualised flow in one of the three models were available and the calculated flow patterns in this model were seen to agree well with the images. Strong impingements of incoming main flows against aneurysmal necks were observed in all models regardless of the bifurcation angle and direction of the aneurysmal protrusion. These impingements presumably caused haemolysis, with ADP originating from haemolysed red blood cells inducing platelet aggregation. Dispersion of flow paths was observed only in the largest model and, consequently, fluid motion was slower than in the other two models. Thus, platelet aggregation was considered to be more active in the largest model. Validity of this discussion was confirmed by application of a platelet aggregation model, which had been proposed in the authors' previous study. It was concluded that the volume of the aneurysmal dome had a significant influence on formation of a low-speed region, which is held to be responsible for active platelet aggregation. Geometric features such as the bifurcation angle and direction of aneurysmal protrusion are considered to be secondary factors contributing to active platelet aggregation.

Keywords: cerebral aneurysm, rupture, haemodynamics, computational fluid dynamics platelet aggretation thrombus formation
\end{abstract}

\section{INTRODUCTION}

When a cerebral artery aneurysm ruptures, it results in a life-threatening subarachnoid haemorrhage, and although not all aneurysms rupture, it is difficult to predict precisely which are likely to do so. Ujiie et al. [1] reported a significant correlation between the probability of rupture and aspect ratio (AR) of aneurysmal dome depth to neck width: almost $80 \%$ of ruptured aneurysms had ARs higher than 1.6. Similar comparisons of ruptured and unruptured aneurysms were made by other researchers such as Weir et al. [2] and Nader-Sepahi et al. [3], who concurred with the correlation between AR and the probability of rupture. Some aneurysms, however, rupture even if their AR is lower than 1.6 and it is untrue that those with an AR higher than 1.6 always rupture. Due to this uncertainty, it is still dangerous to rely only on ARs for rupture prediction. For better rupture prediction, more understanding of the haemodynamics and physiology of cerebral aneurysms is necessary.

According to a theory proposed by Ujiie et al. [4], thrombogenesis is likely in aneurysms with high ARs due to extremely slow blood flow therein. Ensuing fibrinolysis in which thrombi are dissolved is considered to cause damage to the aneurysmal walls. In this context, the AR can be interpreted as a parameter which represents the degree of slowness of the blood flow within the aneurysmal dome and, consequently, the likelihood of thrombogenesis. The focus of Ujiie et al.

(C) 2010 WIT Press, www.witpress.com

ISSN: 1755-7437 (paper format), ISSN: 1755-7445 (online), http://journals.witpress.com

DOI: 10.2495/DNE-V5-N2-122-141 
upon ARs seems reasonable because a narrow entrance to an aneurysm prevents kinetic energy from being actively transferred from the main arterial blood flow to the inside of the aneurysm and because greater dispersion of kinetic energy is promoted in larger aneurysms. In an experimental study by Takahashi et al. [5], evidence supporting Ujiie's theory was shown: canine blood flows in endothelialised model aneurysms made of glass were visualised and thrombogenesis was clearly observed in a model aneurysm with an AR of 2.3.

Ujiie's theory has been also supported through modelling. In the previous study [6], the authors tested a model aneurysm identical to one of the glass models in which thrombogenesis was observed in Takahashi's experiment. Assuming platelet aggregation in aneurysms is induced by adenosine diphosphate (ADP), the authors proposed a platelet aggregation model which mathematically describes the physiological reactions of platelets to ADP. This aggregation model was applied to the flow in the model aneurysm after instantaneous velocity profiles in the model had been calculated using an unsteady Navier-Stokes solver. The computational results clearly predicted aggregation of platelets along the aneurysmal wall where the blood flow was considerably slow. Followed by coagulation, active platelet aggregation is considered to lead to a great degree of thrombogenesis.

Although past studies suggest that Ujiie's theory regarding the process of rupture is promising, more detailed study of blood flow within aneurysms is necessary. Approaches based on numerical techniques such as computational fluid dynamics (CFD) are expected to be effective in the investigation of haemodynamic phenomena of which length-scales are small (say $1 \mathrm{~mm}$ or less).

In the previous study [6], it was also demonstrated that the flow pattern in an aneurysmal dome closely correlated with the location of platelet aggregation. It is important, therefore, to classify blood flow patterns into groups by investigation of a number of geometrically different aneurysms. Although a variety of CFD applications to blood flows in cerebral aneurysms have been reported [7-9], blood flow patterns in cerebral aneurysms were not discussed from a viewpoint of likelihood of thrombogenesis or platelet aggregation: those authors focused on parameters in dynamics such as wall shear stress and pressure. Indeed, wall shear stress and pressure are expected to play an important role in the process of aneurysmal growth, but more attention should be paid to the extent of correlation between the degree of thrombogenesis and the risk of rupture.

In this study, unsteady blood flow simulations with unstructured grids were conducted for three different model aneurysms in order to compare flow patterns and their impact on the likelihood of platelet aggregation. Details of the three model aneurysms are described in Section 2. Computational techniques are explained in Section 3, computational results are discussed in Section 4.

\section{MODEL ANEURYSMS}

Because it is known that a great number of cerebral aneurysms are found at bifurcations where anterior communicating arteries separate from anterior cerebral arteries, three models of aneurysms at cerebral artery bifurcations were investigated. The three models had comparable ARs ranging between 2.0 and 2.3 so that the influence of factors other than AR could be investigated. Note that each model had an AR of over 1.6, which equals to aneurysms with potential risk of rupture.

The important parameters of the fluid dynamics are summarised in Table 1. Reynolds numbers were based on inlet pipe diameter $(D)$ and mean velocity at the inlet $\left(u_{m}\right)$. The mean velocity was calculated from

$$
u_{m}(t)=\frac{\int_{A} w d A}{\int_{A} d A}=\frac{4 Q(t)}{\pi D^{2}}
$$


Table 1: Important parameters of the geometry, fluid dynamics and computational conditions.

\begin{tabular}{llll}
\hline & \multicolumn{1}{c}{ Model 1 } & \multicolumn{1}{c}{ Model 2 } & \multicolumn{1}{c}{ Model 3 } \\
\hline Reynolds number & Min.244 Max.485 & Min.399 Max.797 & Min.366 Max.728 \\
Womersley number & 2.39 & 2.40 & 2.25 \\
Aspect ratio (AR) & 2.3 & 2.0 & 2.0 \\
Flow rate curve & Fig. 2 (measured) & Sinusoidal & Fig. 2 \\
Reference experiment & Takahashi et al. [5] & Shimamura [11] & N/A \\
Computational cells & 886,893 & $1,127,077$ & $1,220,816$ \\
\hline
\end{tabular}

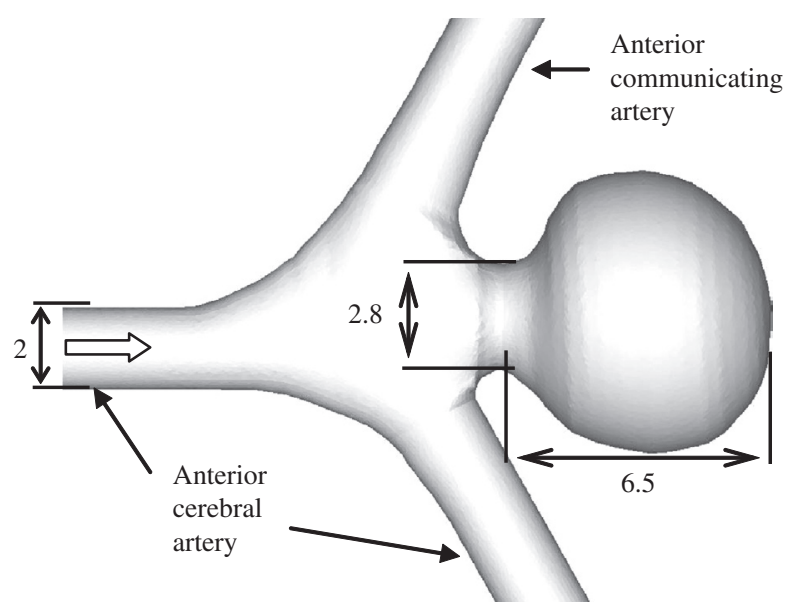

Figure 1: The geometry of Model 1 (all dimensions in $\mathrm{mm}$ ).

where $w$ and $Q$ denote the incoming velocity component and flow rate, respectively. Due to the pulse, the flow rate and mean velocity displayed temporal variation. $Q$ and $u_{m}$, therefore, represent instantaneous values of the respective parameters. The minimum and maximum Reynolds numbers shown in Table 1 were based on $u_{m, \max }=\max \left(u_{m}\right)$ and $u_{m, \min }=\min \left(u_{m}\right)$, respectively. Womersley numbers were defined by inlet pipe radius and pulsation frequency.

Details of the geometry and computational conditions are explained in the following sections.

\subsection{Model 1}

Model 1 is shown in Fig. 1 where a thick arrow indicates the direction of incoming flow. The aneurysmal dome was located at a Y-shaped bifurcation. Each pipe was straight and $2 \mathrm{~mm}$ in diameter. The whole model was symmetrical about the central plane, which is, in Fig. 1, parallel to the surface of the page. Model 1 was identical to one of the glass aneurysms experimentally tested by Takahashi et al. [5]. Their original glass model was handcrafted and its fine geometric details could not be determined beforehand. The geometric data of Model 1 was, therefore, obtained manually from several digital images of the glass model. For ease of acquisition of geometric data, the glass model 


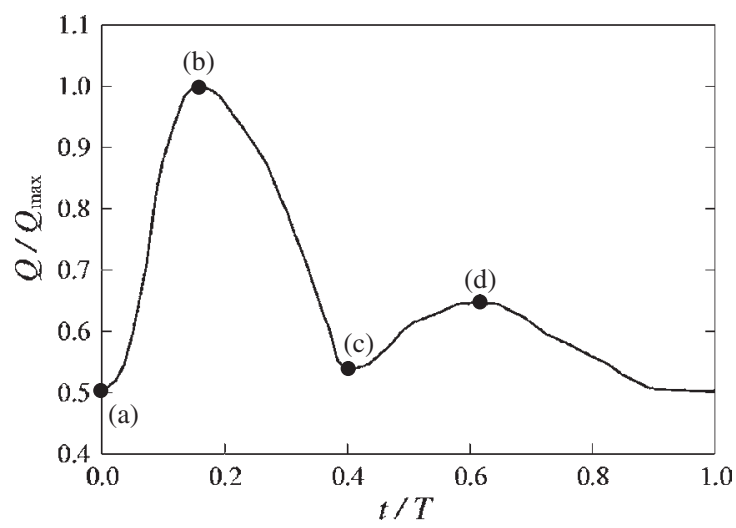

Figure 2: The curve of flow rate imposed at the inlets of Models 1 and 3. $Q_{\max }$ and $T$ stand for the maximum flow rate and pulsation period, respectively.

was cut in half and put on a piece of paper ruled into $1 \mathrm{~mm}$ squares. The depth of the aneurysmal dome and the neck width measured 6.5 and $2.8 \mathrm{~mm}$, respectively, with an AR of 2.3.

During the experiment by Takahashi et al. [5], they recorded the instantaneous flow rate $Q(t)$. In this numerical study, the inlet boundary conditions were derived from their flow rate curve, which is shown in Fig. 2. Indices (a)-(d) in Fig. 2 represent time points of extrema. The period of the cyclic change, $T$, was $0.276 \mathrm{~s}$ and the Womersley number amounted to 2.39 . The Reynolds number was at its lowest at point (a) and highest at (b), ranging between 244 and 485 .

When inlet velocity profiles were calculated from the flow rate curve in Fig. 2, fully developed axisymmetric flow with no swirl was assumed. A profile of the incoming velocity component $w$ was obtained as a function of radius $r$ by solving the following set of equations at each time step:

$$
\begin{gathered}
\rho \frac{\partial w}{\partial t}=-\frac{d p}{d z}+\frac{1}{r} \cdot \frac{\partial}{\partial r}\left(\mu r \frac{\partial w}{\partial r}\right) \\
2 \pi \int_{0}^{R} w r d r=Q(t)
\end{gathered}
$$

where $d p / d z, \rho$ and $\mu$ stand for pressure gradient, density and viscosity, respectively. Equation (2) is the equation of motion in the cylindrical coordinate system while eqn (3) expresses the flow rate condition. When the system of the equations was solved, the pressure gradient was treated as an unknown. Firstly, eqn (2) was solved with an initial approximation to $d p / d z$. Secondly, $d p / d z$ was updated based on eqn (3) and also velocity components were corrected. These two steps of computation were repeated until both equations were satisfied. This solution process is similar to the SIMPLE method [10].

At the bifurcation, $40 \%$ of blood proceeded to the anterior communicating artery and the rest continued flowing in the anterior cerebral artery. This ratio of the outgoing flow rates was constant throughout the cycle. 


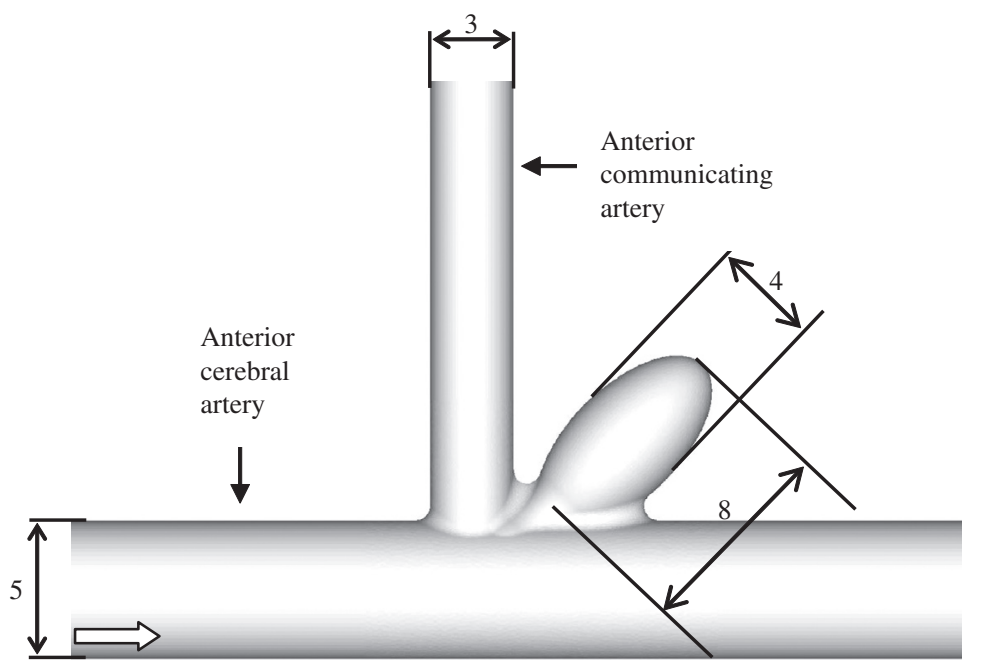

Figure 3: The geometry of Model 2 (all dimensions in $\mathrm{mm}$ ).

\subsection{Model 2}

Model 2 was originally designed by Shimamura [11] who visualised the flow pattern in the dome. He made up the model by rapid prototyping. In this study, the authors reproduced the water flow in Shimamura's experiment using numerical techniques.

As shown in Fig. 3, Model 2 mimicked an ellipsoidal aneurysm at a T-shaped cerebral artery bifurcation where two arteries met at a right angle. Like Model 1, the whole model was symmetrical about the central plane. The main pipe (anterior cerebral artery) and branch (anterior communicating artery) were 5 and $3 \mathrm{~mm}$ in diameter, respectively. The diameters of these pipes in Model 2 were increased to exceed those typical of real human cerebral arteries for ease of visualisation of flow.

The aneurysmal dome inclined by 45 degrees. Its major and minor axes are 8 and $4 \mathrm{~mm}$ long, respectively. It is difficult to determine depth and neck width of the aneurysm precisely because the aneurysm was partially embedded within the two arteries. However, the length of the major axis, 8 $\mathrm{mm}$, seems a good approximation to the dome depth and, similarly, the minor axis can be used instead of the neck width. Using these approximations, the AR was determined to be 2.0.

Generally, it is difficult in experiment to generate a flow rate curve which precisely mimics a real human pulsation curve. Therefore, a sinusoidal curve of the inlet flow rate was used in Shimamura's experiment. In this numerical study, inlet boundary conditions based on the same sinusoidal flow rate curve were imposed. Detailed velocity profiles at the inlet were calculated from the system of eqns (2) and (3) in the same manner as in Model 1.

In Shimamura's experiment, water was used instead of blood while the model aneurysms and arteries were larger than actual ones. According to the similarity law, flow features observed in such a model would be irrelevant to actual blood flow unless Womersley and Reynolds numbers in the model agreed with those in a real human cerebral artery. Therefore, the Womersley number and minimum and maximum Reynolds numbers were set at 2.40, 399 and 797, respectively, all of which were considered to be realistic values. 


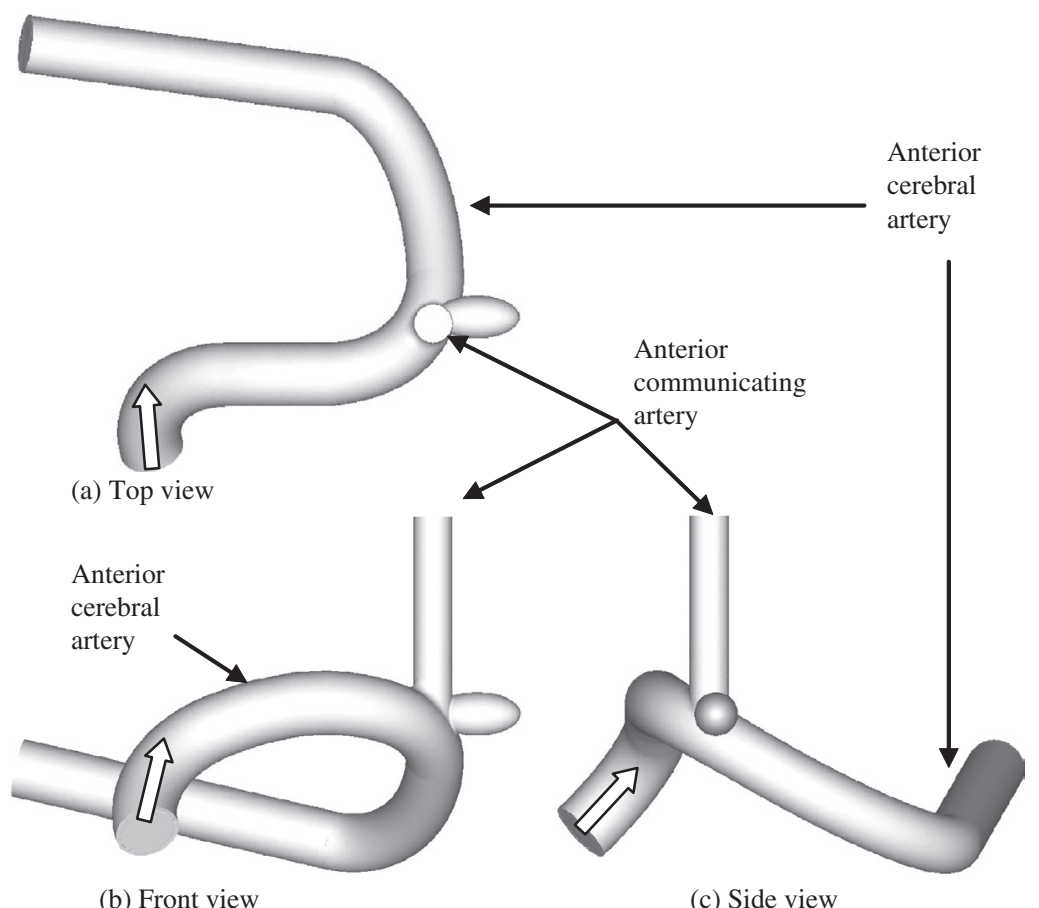

Figure 4: Views of Model 3 in three directions.

It was confirmed in Shimamura's experiment that, throughout the cycle, $26 \%$ of the incoming blood moved to the branch at the bifurcation.

\subsection{Model 3}

Models 1 and 2 had 3-dimensional geometry, but arteries in the two models were mimicked using simple straight pipes. This is unrepresentative of real cerebral artery bifurcations. The authors, therefore, tested Model 3 with more realistic features.

Model 3, shown in Fig. 4, consisted of a 3-dimensionally curved artery, a straight branch and an ellipsoidal aneurysm. The direction of protrusion of the dome was determined according to [12] in which the most likely features of anterior communicating artery aneurysms were mentioned. In Model 3, the anterior cerebral artery shrank a little: the diameter measured $3 \mathrm{~mm}$ at the inlet and 2.7 $\mathrm{mm}$ after the bifurcation. The branch (anterior communicating artery) was $1.8 \mathrm{~mm}$ in diameter. The dome was an ellipsoid with major and minor axes of 4 and $2 \mathrm{~mm}$, respectively. The AR was determined to be 2.0 in the same manner as in Model 2.

A flow rate curve similar to that used for Model 1 was imposed when determining the inlet boundary conditions. The Womersley parameter was 2.25 . The maximum and minimum Reynolds numbers at the inlet were 366 and 728, respectively. Thirty percent of the blood flowed into the anterior communicating artery.

\section{COMPUTATIONAL TECHNIQUES}

The unsteady incompressible Navier-Stokes equations (4) and continuity equation (5) were numerically solved. 


$$
\begin{gathered}
\frac{\partial\left(\rho u^{i}\right)}{\partial t}+\frac{\partial\left(\rho u^{j} u^{i}\right)}{\partial x^{j}}=-\frac{d p}{d x^{i}}+\frac{\partial \tau^{j i}}{\partial x^{j}} \quad(i=1,2,3) \\
\frac{\partial u^{j}}{\partial x^{j}}=0
\end{gathered}
$$

where $\rho, \mu$ and $u^{i}$ stand for fluid density, viscosity and the Cartesian component of velocity vector in the $x^{i}$ direction, respectively. Einstein's summation rule is applied to these equations and $j$ is a dummy index. $\tau^{j i}$ is the component of viscous stress tensor associated with the strain rate through the following equation:

$$
\tau^{j i}=\mu\left(\frac{\partial u^{i}}{\partial x^{j}}+\frac{\partial u^{j}}{\partial x^{i}}\right)
$$

Note that eqn (4) is expressed in the conservation form for ease of application of the finite volume method (FVM). Flow was assumed to be laminar in all model aneurysms.

The SIMPLE algorithm [10] was employed for pressure-velocity linkage. The cell-centred finite volume approach in which all unknowns are defined at the centre of each computational cell was used for spatial discretisation of the governing equations. The cell-centred arrangement of unknowns is likely to cause a physically meaningless oscillation in calculated pressure fields. This error in solutions, known as ‘spurious error', was avoided via Rhie and Chow's method [13].

Unstructured grid systems were employed so that the complicated geometry of each model could be properly expressed. In unstructured grid systems, each computational cell is usually in the shape of a tetrahedron, triangular prism, pyramid or hexahedron. In this study, mainly tetrahedral cells were used. However, tetrahedral cells seemed undesirable for boundary layer flows which were parallel to wall surfaces and intersected surfaces of tetrahedral cells at oblique angles. This would lead to a loss of accuracy in the solution [10] and instability of calculation. Therefore, hexahedral and prismatic cells were allocated in near-wall regions.

The number of computational cells was 886,893 in Model 1, 1,127,077 in Model 2 and 1,220,816 in Model 3. The time dependent term in the L.H.S of eqn (4) was approximated by the 3rd order backward difference scheme and its treatment was fully implicit. For Model 1, two settings of time step $\Delta t$ were tested: $\Delta t=T / 2760$ and $T / 500$, where $T$ represents the pulsation period. It was confirmed that there was no significant difference between the two solutions. The computational results for Model 1 presented in Section 4 are those obtained with $\Delta t=T / 2760$. Considering experiences with Model 1, the authors determined the time step $\Delta t$ for the other models: T/500 for Model 2 and $T / 1000$ for Model 3. A longer time step was used for Model 2 because, as explained in Section 2, the inlet flow rate in Model 2 was expressed by a sinusoidal curve which was free from any rapid increase or decrease.

The authors' original CFD code was used in solution of the N-S equations. The domain decomposition technique [14] was applied in order to enable parallel computing. The $\mathrm{N}-\mathrm{S}$ solver was implemented on a PC cluster with sixteen Pentium-4 processors. The whole computational domain was partitioned into eight subdomains in Model 1 while the number of subdomains in Models 2 and 3 was sixteen. Connecting boundaries between neighbouring subdomains were treated with the overlap cell technique [15] in which computational cells along connecting boundaries are shared by the two corresponding subdomains. Communication between processors was provided by MPI 
(message passing interface). In the parallelisation strategy, processors communicated with each other every time unknowns were updated so that any slowdown in convergence speed could be minimised [14].

Blood was assumed to be a Newtonian fluid in Models 1 and 3 while water flow was analysed in Model 2. Viscosity $\mu$ was, therefore, fixed in all calculations. However, as blood is actually a nonNewtonian fluid, it seemed necessary to examine the influences of the Newtonian assumption on calculated flow patterns. The Carreau-Yasuda model [16] was applied to Model 1 so that Newtonian and non-Newtonian solutions could be compared. In the Carreau-Yasuda model, viscosity $\mu$ is a function of strain rate $\dot{\gamma}$ as follows:

$$
\mu=\mu_{\infty}+\frac{\mu_{0}-\mu_{\infty}}{\left\{1+(\lambda \dot{\gamma})^{b}\right\}^{a}}
$$

Values of the parameters in eqn (7) are shown in Table 2. Note that

$$
\lim _{\dot{\gamma} \rightarrow 0} \mu=\mu_{0}, \quad \lim _{\dot{\gamma} \rightarrow \infty} \mu=\mu_{\infty},
$$

The relation between viscosity $\mu$ and strain rate $\dot{\gamma}$ is shown in Fig. 5. Before the non-Newtonian calculation was carried out, velocity profiles at the inlet had been calculated from the system of eqns (2), (3) and (7).

Table 2: Model parameters used in the Carreau-Yasuda model.

\begin{tabular}{lcccc}
\hline$\mu_{0}$ & $\mu_{\infty}$ & $a$ & $b$ & $\lambda$ \\
\hline $0.16 \mathrm{~Pa} \mathrm{~s}$ & $3.5 \times 10^{-3} \mathrm{~Pa} \mathrm{~s}$ & 1.23 & 0.64 & $8.2 \mathrm{~s}$ \\
\hline
\end{tabular}

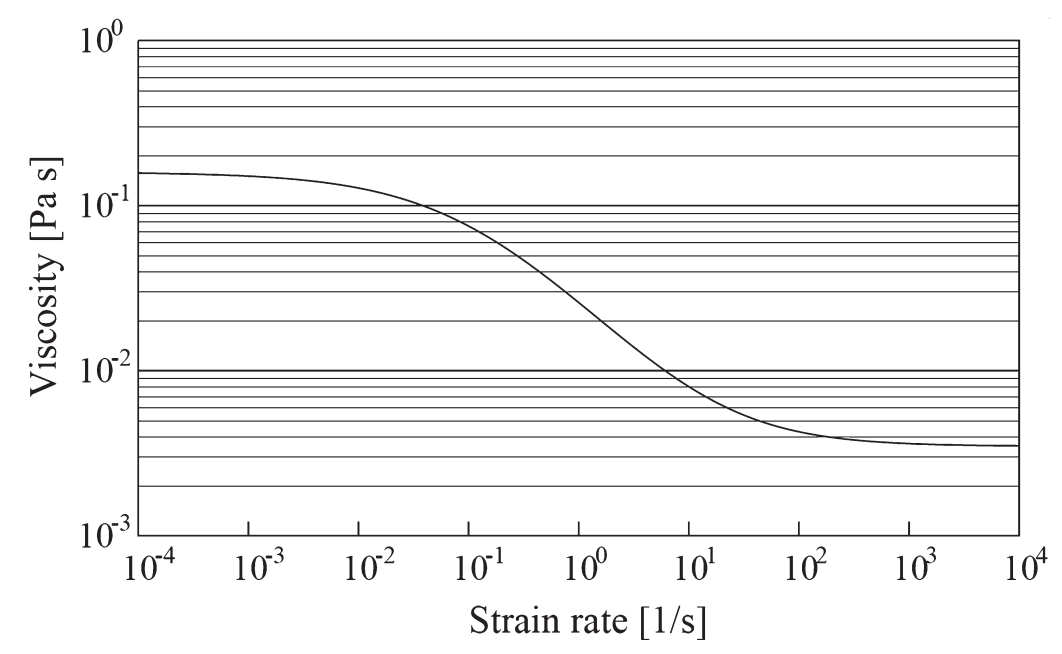

Figure 5: A non-Newtonian relation between viscosity and strain rate expressed by the CarreauYasuda model. The Carreau-Yasuda model was applied only to Model 1. 
The time-marching process in unsteady incompressible flow calculation is mathematically equivalent to serial application of a non-linear vector mapping with a set of initial profiles of velocity and pressure, which usually contain physically meaningless components, and behaviours of the solution are determined by eigen values of the mapping. The non-physical components in the initial profiles continue to decline as the time-marching proceeds because they correspond to eigenvalues of which absolute values are less than unity [17] while eigenvalues of periodic fluctuations such as pulsatile blood flow correspond to unity [18]. However, solutions do not precisely reflect actual flow at an early stage until the non-physical components have become negligible. It is important for reduction in computational time to give the best possible initial profiles because the duration of unreliable early solutions depends on the quality of the initial profiles. In this study, initial profiles were obtained in the following way: a steady problem was solved with a flow rate identical to the highest flow rate of the corresponding unsteady problem. Subsequently, the unsteady flow calculation started at the moment of the highest flow rate with the steady flow solution used as the initial condition. This approach was based on a report by Wahap et al. [19], who found that calculated steady flow in an aneurysmal sac was close to the instantaneous flow in the corresponding unsteady problem at the moment of the highest flow rate. In every model, solutions agreed with those in the previous cycle after approximately 1.5 times the pulsation period had elapsed since the start of the time-marching.

\section{RESULTS AND DISCUSSION}

\subsection{Model 1}

Figure 6 shows velocity vectors on the central cross-sectional plane in Model 1 at the four time points (a)-(d), which are defined in Fig. 2. Each reference arrow in Fig. 6 represents 50\% of the maximum mean velocity $u_{m, \max }$, where $u_{m, \max }=0.97 \mathrm{~m} / \mathrm{s}$. All results in Fig. 6 were obtained on the assumption of a Newtonian fluid.

At each time point, the incoming flow approaching the entrance to the aneurysm from the left changed its direction slightly downward because the outgoing flow rate was higher in the anterior cerebral artery than in the anterior communicating artery. It can be clearly observed that the incoming flow hit the lower edge of the aneurysmal neck. More precisely, the flow dramatically decelerated near the apex of the aneurysmal neck. It is noteworthy that this rapid reduction in flow speed occurred across rather short distance in the vicinity of the neck. As a result, static pressure near the neck rapidly increased because, in accordance with Bernoulli's law, deceleration in flow causes an increase in static pressure. In fact, the apex of the neck was a stagnation point where the highest static pressure was recorded. The pressure gradient near the neck amounted to $0.25 \mathrm{~Pa} / \mu \mathrm{m}$ at temporal point (a) and $0.80 \mathrm{~Pa} / \mu \mathrm{m}$ at (b). As the characteristic length scale of a red blood cell (RBC) is $8 \mu \mathrm{m}$, the pressure difference exerted on RBCs in the impingement region ranged approximately between 2.0 and 6.4 Pa. This pressure difference is considered to be large enough to cause haemolysis with Alkhamis et al. [20] having reported that ADP originally contained in RBCs escaped into plasma when a shear stress of only 2 or $3 \mathrm{~Pa}$ was exerted on RBCs. Here, the term 'haemolysis' does not mean destruction of RBC membranes but is used to express escape of RBC contents through the membranes into the surrounding plasma [21]. Although modes of RBC membrane deformation were different (compression in the present study and shearing in Alkhamis's), levels of strain were presumably comparable.

In addition, a dramatic rise in shear stress was observed immediately after the impingement. The highest wall shear stress was found at the point on the narrowest portion of the aneurysmal neck, 


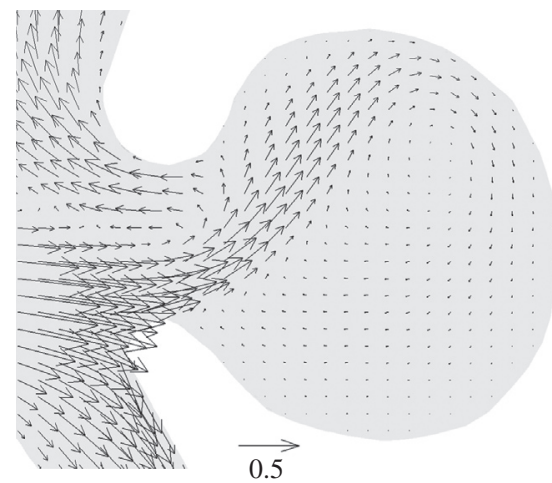

(a) $t / T=0$ (at the lowest flow rate)

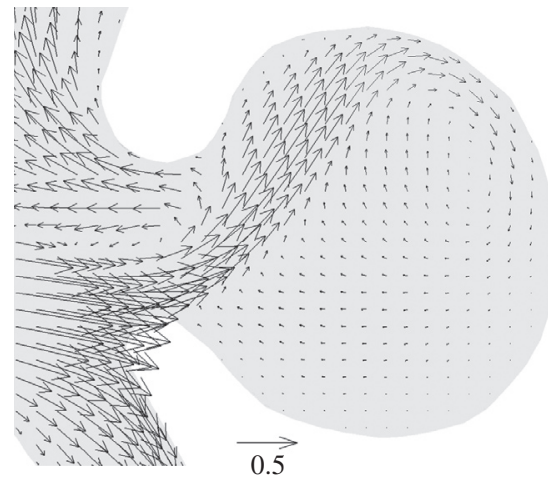

(c) $t / T=0.40$

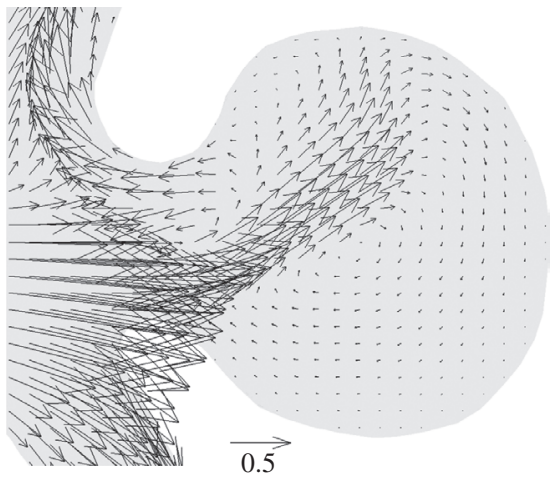

(b) $t / T=0.16$ (at the highest flow rate)

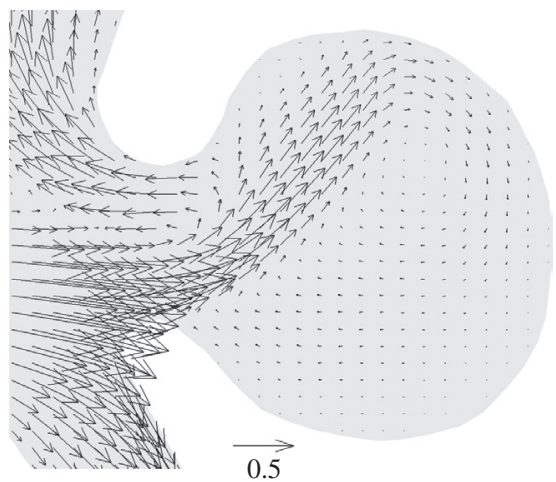

(d) $t / T=0.61$

Figure 6: Calculated velocity vectors on the central plane in Model 1 at four time points with the Newtonian assumption. Each point in time is represented by the ratio of the time to the pulsation period $T$.

which was only $0.5 \mathrm{~mm}$ distant from the stagnation point along the wall. The wall shear stress at this point ranged between 14 and $32 \mathrm{~Pa}$ with its average being $20 \mathrm{~Pa}$. It can be, therefore, concluded that RBCs sequentially received two types of force near the stagnation point:

1. compressing force caused by pressure gradient before the impingement;

2. shearing force due to velocity gradient after the impingement.

Both are considered to be responsible for haemolysis. This conclusion is supported by a finding in Takahashi et al. [5] that significant numbers of platelets were found in thrombi adhering to the wall of the model aneurysm. Note that the model aneurysm was endothelialised: there was no cause of platelet aggregation other than ADP originating from RBCs.

After the impingement upon the neck, some blood containing ADP proceeded to the aneurysmal dome and reached the upper part of the internal wall of the aneurysm. The exact position that the flow reached depend on the point in time because the flow rate and, therefore, inertia of the flow were 


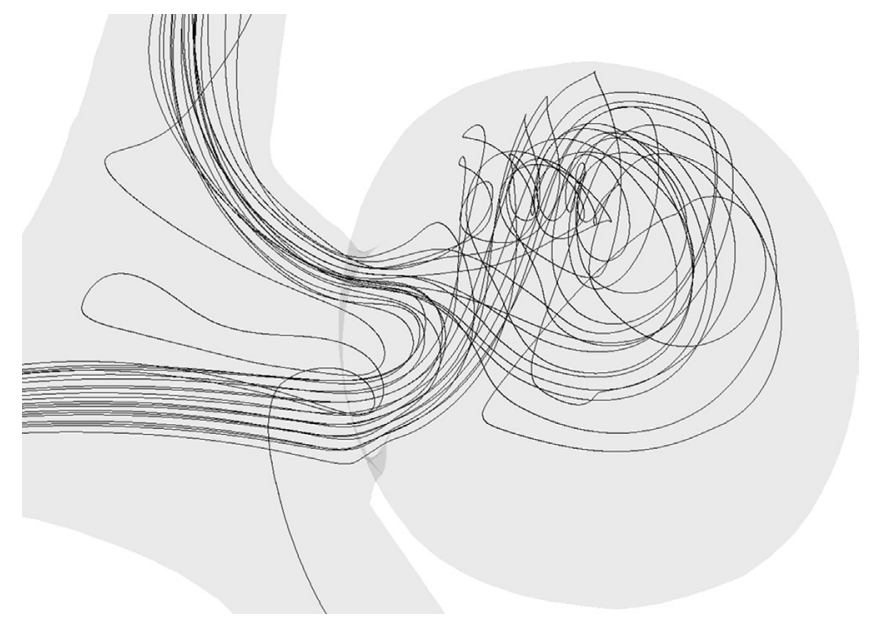

Figure 7: Flow paths in Model 1 (Newtonian). Particles were released at the inlet at $t=0$.

continually changing. The flow, then, changed its direction whilst fanning out in 3-dimensions and dramatically decreasing in speed. A low-speed region was consequently formed and came to occupy a large portion of the dome. The flow velocity was less than $10 \%$ of $u_{m, \max }$ at most points in the lower half of the aneurysm regardless of the timing. The blood motion in the dome after the 3-dimensional fanning was so slow that platelets had enough time to aggregate.

Flow paths within Model 1 are drawn in Fig. 7. In visualisation, imaginary particles were put into the flow at the inlet at temporal point (a). Only the paths of particles which entered the aneurysmal dome are included in Fig. 7 to avoid overlaying too great a number of paths. Flow features in Model 1 such as the impingement upon the neck and the 3-dimensional spread in the dome are clearly observed in Fig. 7. Some particles passing by the neck edge where haemolysis is considered to have occurred stretched across the aneurysmal dome and described long 3-dimensional curves. Blood moving slowly in the dome changed its direction towards the exit and proceeded to the anterior communicating artery.

Takahashi et al. [5] reported that a vortex was visualised in the glass model used in their study. Although no detailed picture of the vortex is included in Reference [5], the authors received the opportunity to have a close look at their video. The shape of the vortex observed in their experiment seemed to agree with the curved path lines in the aneurysm shown in Fig. 7.

Computational results at $t / T=0.230$ are compared in Fig. 8 between (a) Newtonian and (b) nonNewtonian calculations. In each image, the vortex centre (VC) is indicated by the intersection of the horizontal and vertical lines. Difference in the position of VC between the two calculations was $\delta x=0.18 \mathrm{~mm}$ in the horizontal direction and $y_{1}-y_{2}=0.13 \mathrm{~mm}$ in the vertical direction. The position of $\mathrm{VC}$ in the Newtonian calculation was located deeper and lower than in the calculation with the Carreau-Yamada model. This means that relative strength of inertia to viscosity in the Newtonian calculation exceeded that in the non-Newtonian. It follows that this difference resulted from the non-Newtonian characteristic that viscosity increases at a small strain rate. However, this is not of primary importance because the two flow fields showed the same overall features and because the difference in the VC position was less than $10 \%$ of the characteristic length scale (the neck width $2.8 \mathrm{~mm}$ ). 
(a) Newtonian $t / T=0.230$

(b) non-Newtonian $t / T=0.230$

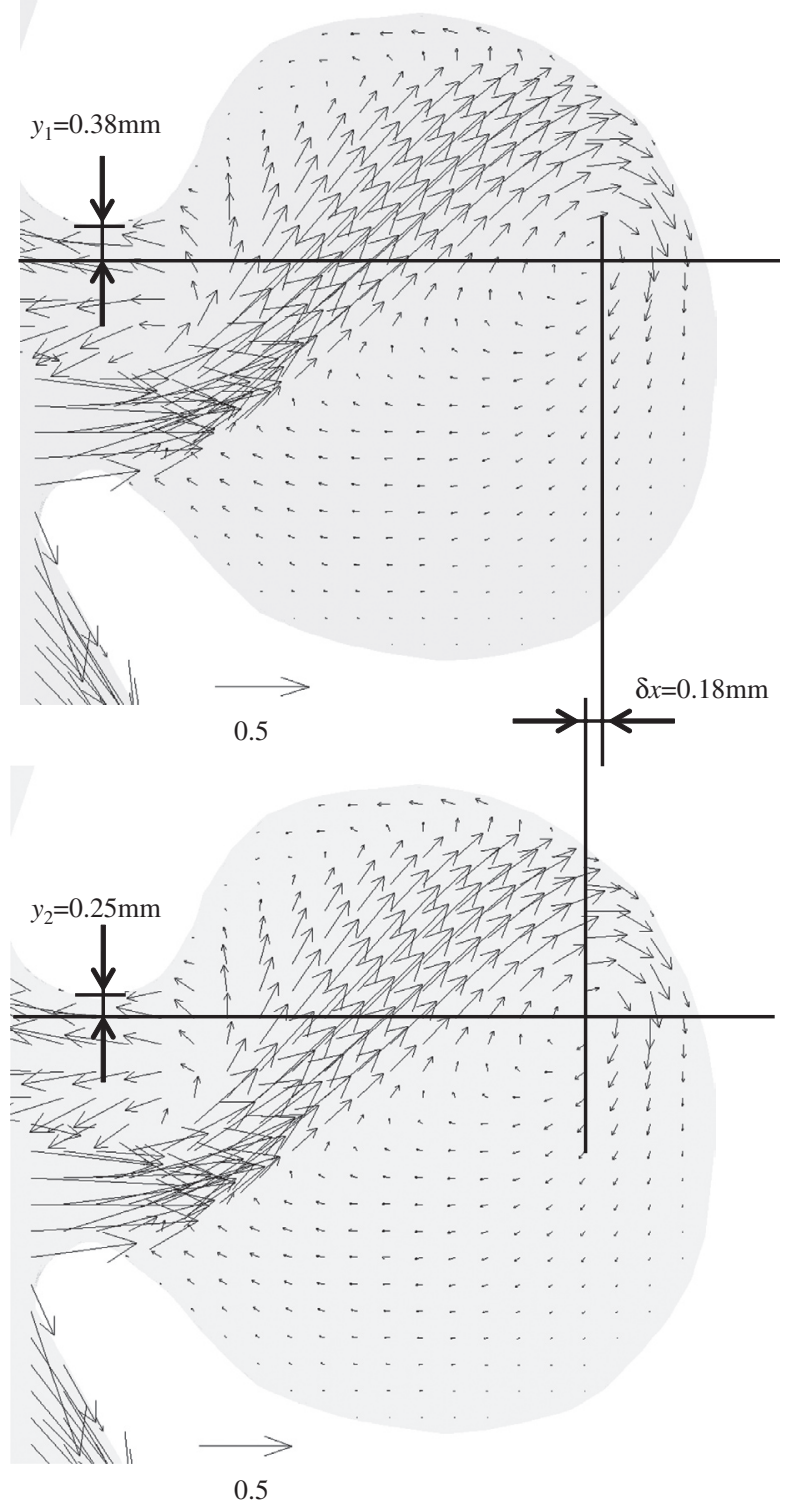

Figure 8: Comparison of vortex centres on the central plane in Model 1 between Newtonian and non-Newtonian calculations. Each snapshot is at $t / T=0.230$.

Figure 9 shows velocity vectors on the central plane obtained with the Carreau-Yasuda model. The four time points are identical to those in Fig. 6. There was no noticeable difference between the Newtonian and non-Newtonian calculations. It is important that the large low-speed region observed in Fig. 6 is also found in Fig. 9 because this low-speed region is considered to be responsible for active platelet aggregation in the aneurysm. It can be concluded from the observations above that assumption of a Newtonian fluid is appropriate to discussion of blood flow patterns in aneurysms. 


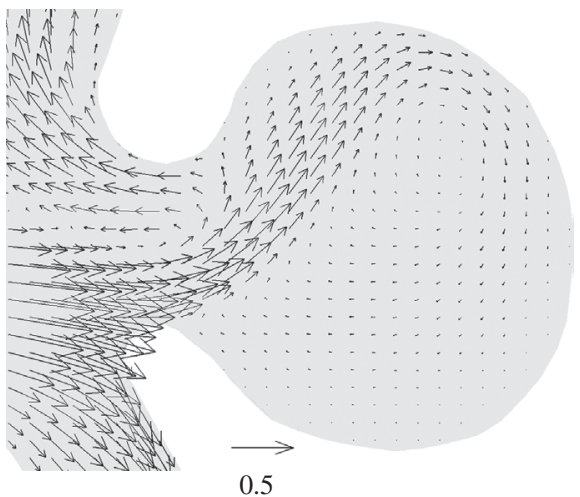

(a) $t / T=0$ (at the lowest flow rate)

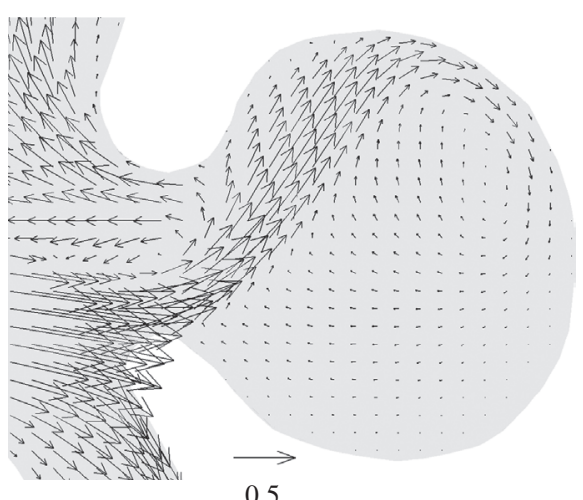

(c) $t / T=0.40$

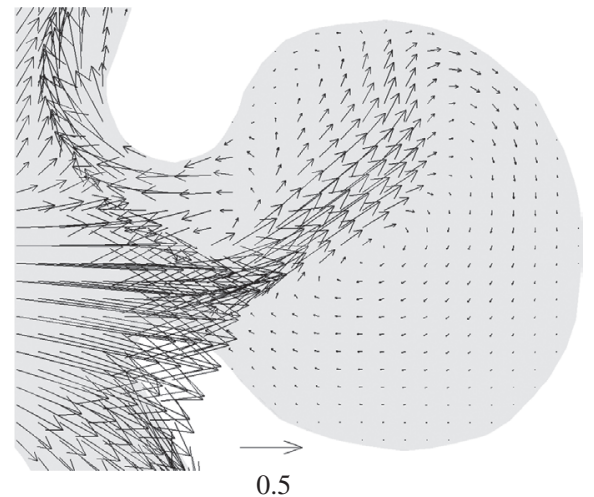

(b) $t / T=0.16$ (at the highest flow rate)

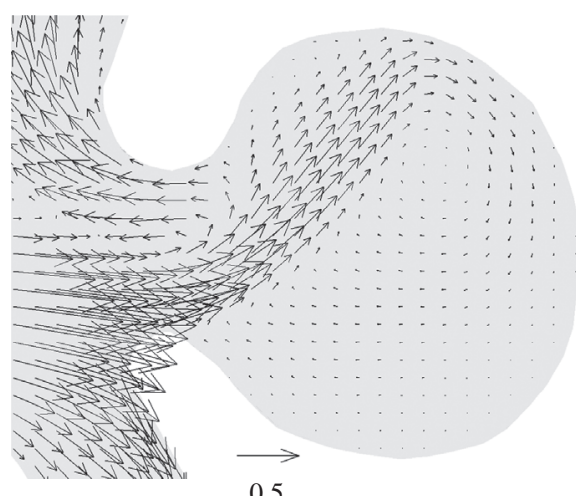

(d) $t / T=0.61$

Figure 9: Calculated velocity vectors on the central plane in Model 1 at four time points with the Carreau-Yasuda. Each point in time is represented by the ratio of the time to the pulsation period $T$.

\subsection{Model 2}

Figure 10 shows velocity vectors on the central plane in Model 2 at four time points. The lowest and highest flow rates came at points (a) and (c), respectively. Acceleration and deceleration of the incoming flow was greatest at (b) and (d), respectively. As the flow rate curve was sinusoidal, the interval of the two neighbouring temporal points was exactly a quarter of the pulsation period. Each reference arrow in Fig. 10 represents $50 \%$ of the maximum mean velocity $u_{m \text {,max }}$, where $u_{m, \max }=0.16 \mathrm{~m} / \mathrm{s}$.

The main incoming stream was drawn upward by suction in the anterior communicating artery. The flow hit the right edge of the aneurysmal neck at each time point. This feature was commonly observed in Models 1 and 2. The flow decelerated before the neck so quickly that a strong pressure gradient was induced. After the impingement, however, the flow went into the dome and changed 


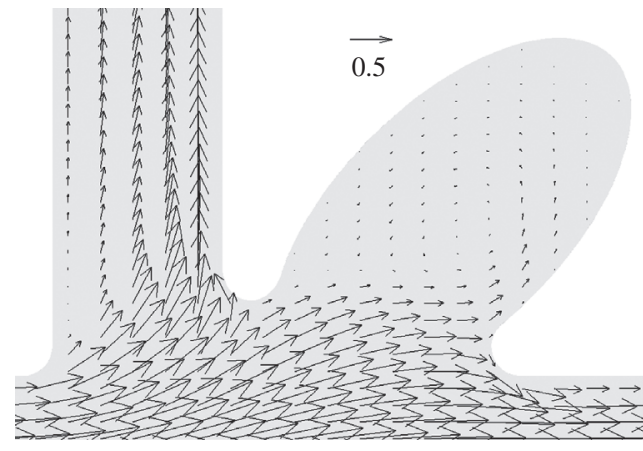

(a) $t / T=0$ (at the lowest flow rate)

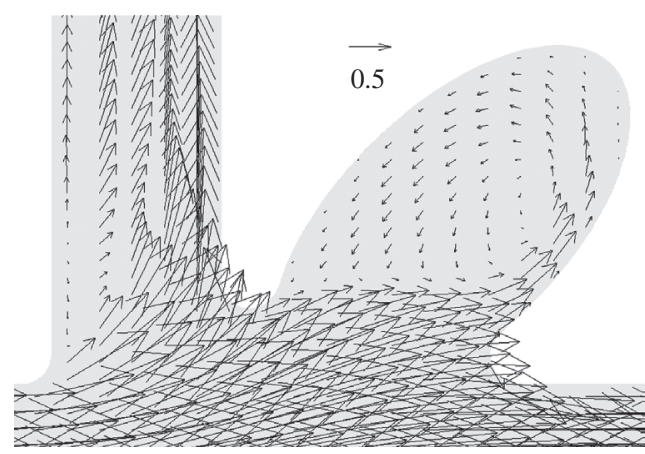

(c) $t / T=0.50$ (at the highest flow rate)

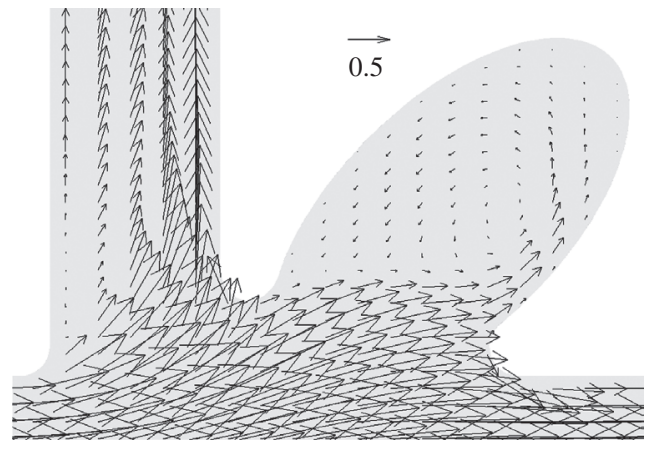

(b) $t / T=0.25$ (in the accelerating phase)

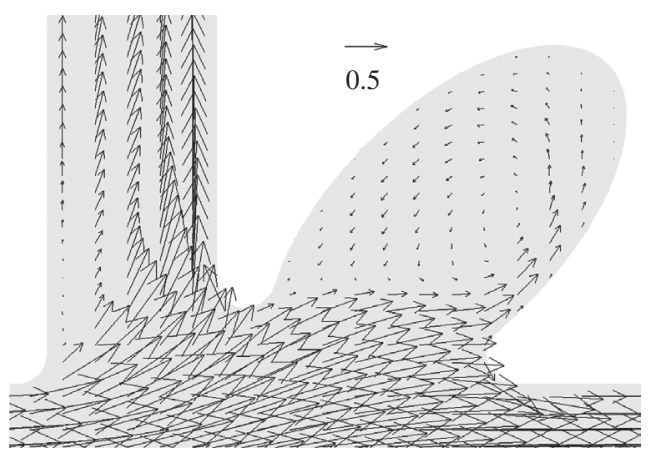

(d) $t / T=0.75$ (in the decelerating phase)

Figure 10: Calculated velocity vectors on the central plane in Model 2 at four time points. Each point in time is represented by the ratio of the time to the pulsation period $T$.

its direction, following the aneurysmal wall in Model 2. This is a sharp contrast to the flow in Model 1, which moved across the aneurysmal dome and kept away from the wall for a period after the impingement (see Fig. 6).

Another important difference between flow patterns in Models 1 and 2 was the magnitude of velocity. As shown in Fig. 6, the lower half of the aneurysm in Model 1 was filled with slowly moving blood of which velocity scale was less than $10 \%$ of $u_{m \text {,max }}$ throughout the cycle. On the other hand, in Fig. 10, the magnitude of the flow velocity in the aneurysmal dome was $10 \%$ of $u_{m \text { max }}$ or more at time points (b)-(d), although the velocity magnitude was less than $10 \%$ at point (a). Thus, the blood entering the aneurysmal dome in Model 2 was quickly flushed out, a pattern not observed in Model 1. Platelet aggregation seems less likely in Model 2 than in Model 1 because platelets tended to exit the aneurysm before aggregation became active.

Figure 11 shows calculated flow paths in Model 2. Flow was visualised in the same manner as in Fig. 7. In Fig. 11, the model is rotated by 45 degrees around the vertical axis so that 3 -dimensional structure of the flow can be seen. After entering the aneurysmal dome, the flow turned round, spreading slightly in the spanwise direction. Upon escaping from the dome in Model 2, the fluid returned to the original main passage and moved along the wall, not on or near the central plane. 


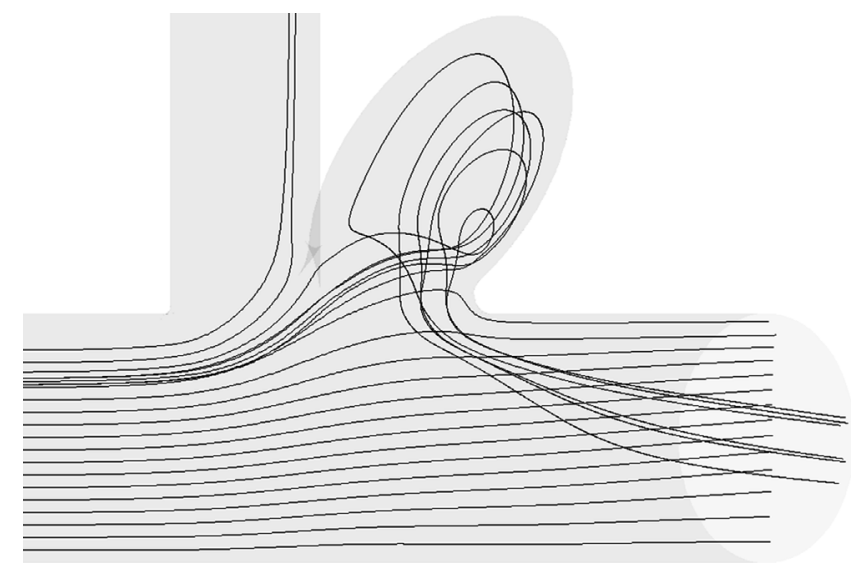

Figure 11: Flow paths in Model 2.

The degree of the spanwise spread in the dome was not great enough to cause the flow to lose much kinetic energy. This can be contrasted with Model 1 where flow paths dispersed within the aneurysm (see Fig. 7).

The calculated flow paths are compared with an experimentally obtained image in Fig. 12. The same flow paths as in Fig. 11 are projected onto the $x-y$ plane in Fig. 12(a). A snapshot from a video recorded in Shimamura's experiment [11] is shown in Fig. 12(b). In his experiment, the flow was visualised by filling the model with milk and the video was taken while the milk was being flushed out. It can clearly be seen that calculated flow paths in and out of the aneurysmal dome correlate well with the wisps of milk in Fig. 12(b).

\subsection{Model 3}

Velocity vectors on the central plane of the aneurysmal dome in Model 3 are drawn in Fig. 13. The view direction of Fig. 13 is identical to that of Fig. 1(a). The four time points, (a)-(d) are defined in Fig. 2. Each reference arrow in Fig. 13 represents $50 \%$ of the maximum mean velocity $u_{m \text {,max }}$, where $u_{m, \max }=0.97 \mathrm{~m} / \mathrm{s}$. An impingement of the mainstream upon the aneurysmal neck is clearly seen in this figure. As, regardless of the bifurcation angles, similar impingements were observed in all models tested in this study, impingements of this kind and the resulting haemolysis are presumably common to most aneurysms located at artery bifurcations.

After the impingement, the flow proceeded along the aneurysmal wall and turned round. This feature was common to Models 2 and 3. Unlike Model 1, no sizable region of extremely slow flow was observed in Model 3. The flow speed was more than $10 \%$ of $u_{m, \max }$ in most part of the aneurysm: the exception being a region close to the deepest end. Therefore, the degree of platelet aggregation in Models 2 and 3 was presumably comparable: platelet aggregation in the two models was less active than in Model 1.

Figure 14 shows flow paths, which were calculated by releasing imaginary particles at the inlet at $t=0$. Flow paths in Model 3 were similar to those in Model 2 because neither of the two models produced flow paths which dispersed within the aneurysmal dome (cf. Figs 11 and 14). This means that blood in the aneurysms lost little kinetic energy whilst maintaining a high flow speed. As a 


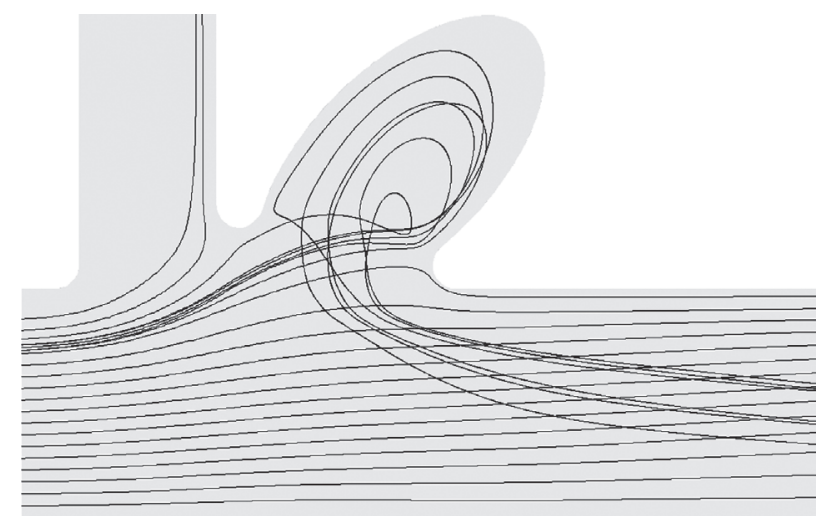

(a) Calculated flow paths (projection onto the $x-y$ plane)

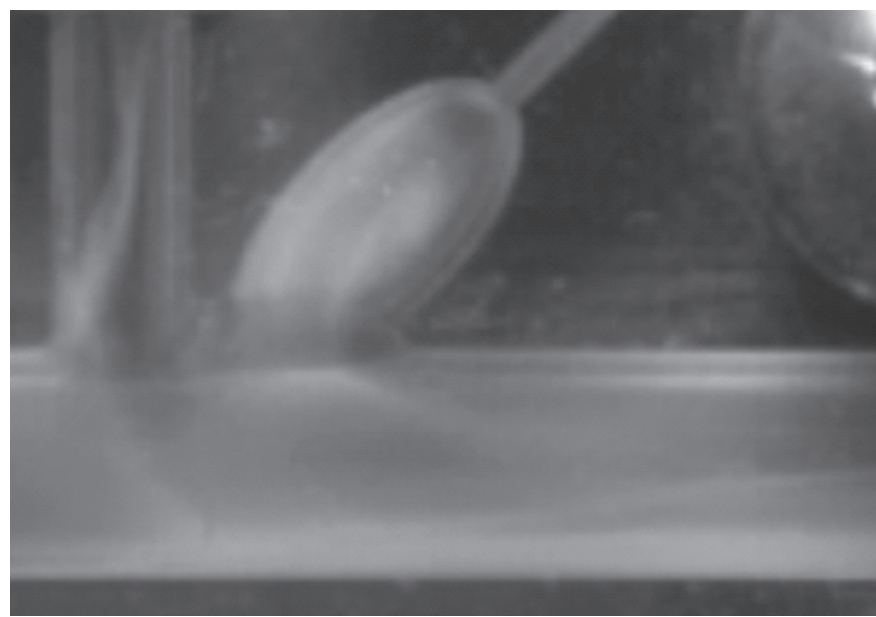

(b) A snapshot during the sweeping process of infused milk. (Courtesy of Tokyo University of Science)

Figure 12: Comparison between (a) calculated and (b) experimentally visualised flow patterns in Model 2.

whole, the flow structure in Model 3 was similar to that in Model 2 although the flow in Model 3 was more 3-dimensional.

\subsection{Likelihood of platelet aggregation}

Every model tested had a chance of haemolysis and ensuing platelet aggregation in its aneurysm because the mainstream always hit the edge of the aneurysmal neck. The flow in Model 1 was characterised by dispersion, which resulted in a large low-speed region. In the other two models, by contrast, no low-speed region was observed. It can be concluded that platelet aggregation was less active in Models 2 and 3 because ADP released from RBCs due to the flow impingement was flushed out more quickly than in Model 1. 


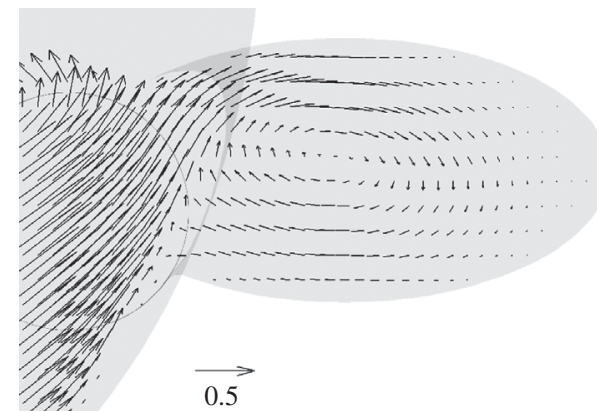

(a) $t / T=0$ (at the lowest flow rate)

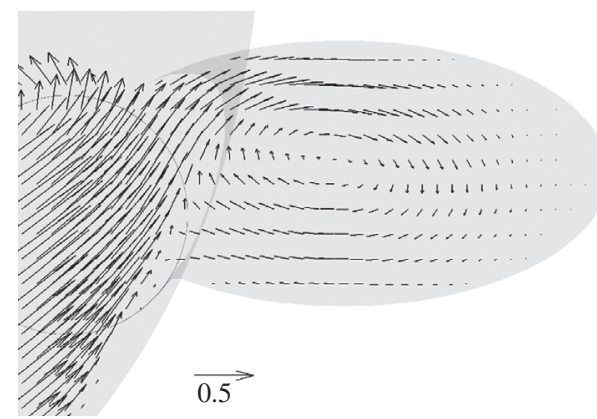

(c) $t / T=0.40$

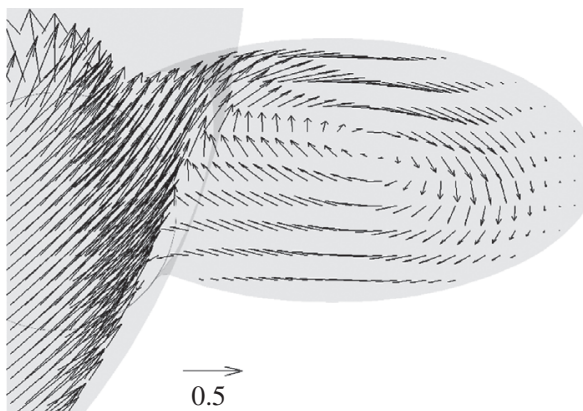

(b) $t / T=0.16$ (at the highest flow rate)

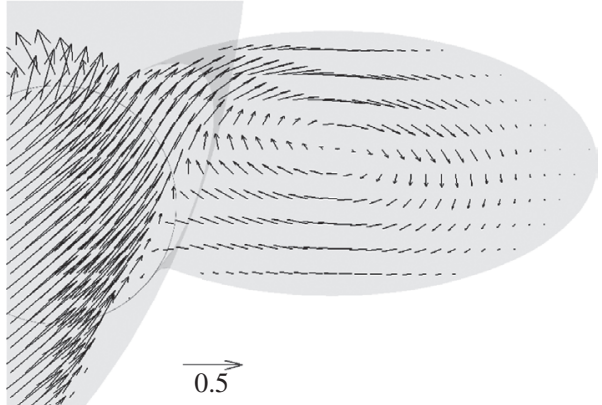

(d) $t / T=0.61$

Figure 13: Calculated velocity vectors in Model 3 at four time points. Vectors are depicted on the central cross-section of the aneurysm and the view direction is identical to that of Fig. 3(a). Each point in time is represented by the ratio of the time to the pulsation period $T$.

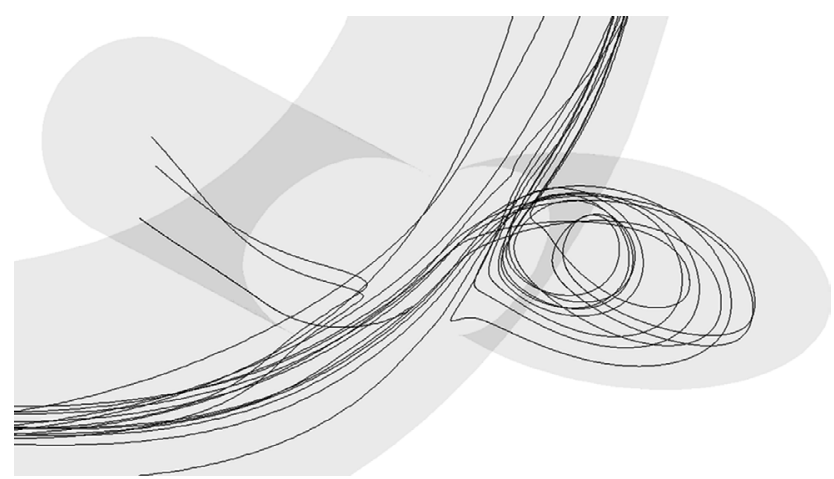

Figure 14: Calculated flow paths in Model 3.

Figures 15 and 16 show calculated distributions of $N_{1}$ (density of aggregated platelets) in the vicinity of aneurysmal walls in Models 1 and 3. Here, the term 'aggregated platelets' is taken to mean those in primary aggregation. Density of aggregated platelets was calculated using the platelet aggregation model [6] on the assumption that $20 \%$ of ADP contained in RBCs was released into plasma in the region within a $1 \mathrm{~mm}$ radius of the stagnation point. Although this estimation of the 


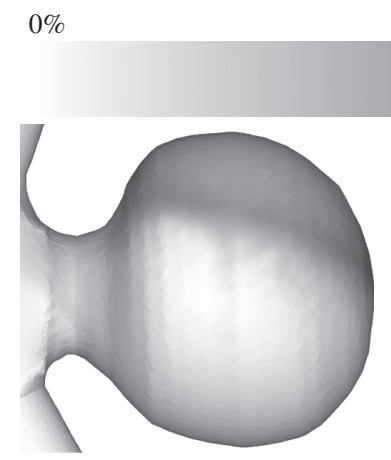

(a) $t / T=4$

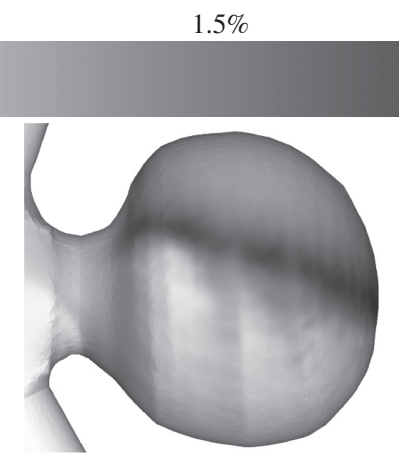

(b) $t / T=8$

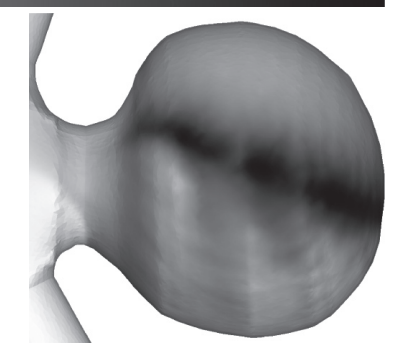

(c) $t / T=12$

Figure 15: Distributions of $N_{1}$ (density of primary-aggregated platelets) in the vicinity of the wall surface in Model 1.

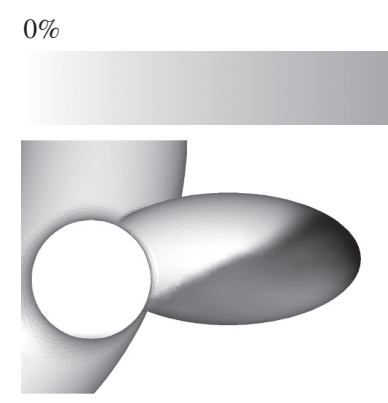

(a) $t / T=1$

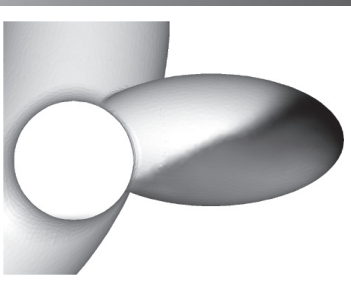

(b) $t / T=6$
$0.4 \%$

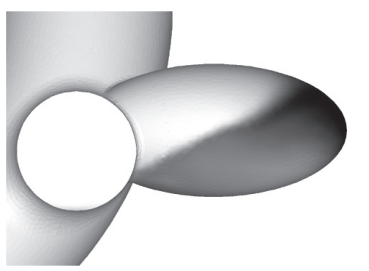

(c) $t / T=11$

Figure 16: Distributions of $N_{1}$ (density of primary-aggregated platelets) in the vicinity of the wall surface in Model 3.

degree of haemolysis was based on the time scale of thrombus formation reported by Takahashi et al. [5], its accuracy has not been ascertained. The following discussion will, therefore, concern only the platelet aggregation tendencies observed in Figs 15 and 16.

It can be seen in Fig. 15(a) that platelet aggregation in Model 1 started from the upper half of the aneurysm because ADP originating from RBCs was first brought to this region by blood flow (see Fig. 6). By the time eight pulse cycles had elapsed, a swathe of high $N_{1}$ had been formed (Fig. 15(b)). The peak value of $N_{1}$ reached $3 \%$ of the whole platelets after 12 pulsation periods (Fig. 15(c)) and continued to increase thereafter. In Model 3, on the other hand, platelet aggregation was mainly observed near the deep end of the aneurysm where the flow was slower. However, platelet aggregation was not found to progress after the first cycle: the maximum value of $N_{1}$ would not exceed $0.8 \%$ of the whole platelets even after 11 cycles. This was due to the high-speed flow in Model 3 where ADP and aggregated platelets were quickly flushed out of the aneurysm. Thus, these results obtained by application of the platelet model support the discussion in the first paragraph of this section.

The AR (2.3 for Model 1 and 2.0 for Models 2 and 3) had an influence on the different flow patterns to some extent because the AR represents the relative size of the aneurysm in the longitudinal direction. 
However, the larger volume of the aneurysmal dome in Model 1 seems to provide a more reasonable explanation for the dispersion and low-speed region therein. The volume of the dome was so large in Model 1 that the mainstream failed to provide fluid in the dome with enough kinetic energy to allow rapid motion. The degree of platelet aggregation is presumably influenced not only by the AR but also the size of the aneurysmal dome in the lateral direction. The bifurcation angle and direction of aneurysmal protrusion seem to be secondary factors contributing to the degree of platelet aggregation because Models 2 and 3 showed similar flow features in spite of different angles of bifurcation and dome protrusion.

\section{CONCLUSIONS}

Unsteady flow simulation was conducted for three different model aneurysms: Model 1 at a Y-shaped bifurcation, Model 2 at a T-shaped bifurcation and Model 3 which represented more realistic anatomical features of cerebral arteries. Impingements of the main flow upon the aneurysmal neck were observed in all the models. This suggests that haemolysis is likely to occur at the entry points of most cerebral aneurysms. A large region filled with low-speed fluid was found in Model 1 while fluid entering the domes was discharged quickly from aneurysms in Models 2 and 3. Therefore, it can be concluded that platelet aggregation was less active in Models 2 and 3. Computational results using an aggregation model supported this conclusion. The difference in flow patterns was attributed to the volume of the aneurysms. The present study suggests that not only AR but also aneurysmal size in the lateral direction should be taken into consideration when the likelihood of platelet aggregation in aneurysms is discussed.

\section{ACKNOLEDGEMENTS}

The authors would like to thank two postgraduates from Tokyo University of Science, Mr Noriyoshi Takahashi and Mr Takashi Shimamura, for providing their experimental data. Mr David $\mathrm{H}$. McMahon's advice on English writing is also gratefully acknowledged.

\section{REFERENCES}

[1] Ujiie, H., Tamano, Y., Sasaki, K. \& Hori, T., Is the aspect ratio a reliable index for predicting the rupture of a saccular aneurysm? Neurosurgery, 48(3), pp. 495-503, 2001. doi:10.1097/00006123-200103000-00007

[2] Weir, B., Amidei, C., Kongable, G., Findlay, J.M., Kassell, N.F., Kelly, J., Dai, L. \& Karrison, T.G., The aspect ratio (dome/neck) of ruptured and unruptured aneurysms. Journal of Neurosurgery, 99(3), pp. 447-451, 2003. doi:10.3171/jns.2003.99.3.0447

[3] Nader-Sepahi, A., Casimiro, M., Sen, J. \& Kitchen, N.D., Is Aspect Ratio A reliable Predictor of Intracranial Aneurysm Rupture? Neurosurgery, 54(6), pp. 1343-1348, 2004. doi:10.1227/01. NEU.0000124482.03676.8B

[4] Ujiie, H., Tachibana, H., Hiramatsu, O., Hazel, A.L., Matsumoto, T., Ogasawara, Y., Nakajima, H., Hori, T., Takakura, K. \& Kajiya, F., Effects of size and shape (aspect ratio) on the hemodynamics of saccular aneurysms: a possible index for surgical treatment of intracranial aneurysms. Neurosurgery, 45(1), pp. 110-130, 1999. doi:10.1097/00006123-199907000-00028

[5] Takahashi, N., Ujiie, H., Yotoriyama, T., Suzuki, Y., Hori, T. \& Kaibara, M., Flow visualization study of the endothelialized glass aneurysm model implanting canine carotid artery (in Japanese with English abs.). Journal of Japanese Society of Biorheology, 18(4), pp. 143-148, 2004.

[6] Shimano, K., Hayashi, T., Ujiie, H., Ono, T. \& Enomoto, Y., Modelling of platelet aggregation in aneurysm, Proc. of 7th Int. Conf. On Modelling In Medicine and Biology, ed. C.A. Brebbia, WIT Press: Southampton, pp. 43-52, 2007. 
[7] Torii, R., Oshima, M., Kobayashi, T., Takagi, K. \& Tezduyar, T.E., Influence of wall elasticity on image-based blood flow simulations (in Japanese with English abs.). Transactions of Japan Society of Mechanical Engineers, Series A, 70(697), pp. 1224-1231, 2004.

[8] Funazaki, K., Higashi, M., Yamada, K., Taniguchi, H. \& Tomura, N., Flow-Structure Coupled Analysis of Cerebrovascular Artery with an Aneurysm of Realistic Geometry (in Japanese with English abs.). Transactions of Japan Society of Mechanical Engineers, Series B, 73(731), pp. 1472-1479, 2007.

[9] Utter, B. \& Rossmann J.S., Numerical simulation of saccular aneurysm hemodynamics: Influence of morphology on rupture risk. Journal of Biomechanics, 40(12), pp. 2716-2722, 2007. doi:10.1016/j.jbiomech.2007.01.011

[10] Patankar, S.V., Numerical Heat Transfer and Fluid Flow, Hemisphere publishing: Washington D.C., pp. 126-131, 1980.

[11] Shimamura, T., A Study on Flow in Aneurysms (in Japanese), Graduation thesis, Tokyo University of Science, Tokyo, 2006.

[12] Satoh, A., Nakamura, H., Kobayashi, S., Miyata, A., Tokunaga, H., Wada, M. \& Watanabe, Y., Surgical approaches and techniques for anterior communicating artery aneurysms: from angioanatomical point of view (in Japanese with English abs.). Surgery for Cerebral Stroke, 30, pp. 240-246, 2002. doi:10.2335/scs.30.240

[13] Rhie, C.M. \& Chow, W.L., Numerical study of the turbulent flow past an airfoil with trailing edge separation. AIAA Journal, 21(11), pp. 1525-1532, 1983. doi:10.2514/3.8284

[14] Shimano, K. \& Arakawa, C., Numerical simulation of incompressible flow on parallel computer with the domain decomposition technique, Parallel Computational Fluid Dynamics: New Algorithms and Applications (Proc. of the Parallel CFD'94 Conference), eds N. Satofuka, J. Periaux \& A. Ecer, Elsevier: Amsterdam, pp. 189-19, 1995.

[15] Shimano, K., Okudera, K., Anaguchi, T., Utsumi, N., Saito, M., Sumie, C. \& Enomoto, Y., Parallel Computing of Flow in Centrifugal Fan Volute Using Contravariant Physical Velocity, Parallel Computational Fluid Dynamics: Multidisciplinary Applications (Proc. of the Parallel CFD 2004 Conference), eds G. Winter, A. Ecer, J. Periaux, N. Satofuka \& P. Fox, Elsevier: Amsterdam, pp. 313-320, 2005.

[16] Leuprecht, A. \& Perktold, K., Computer simulation of non-Newtonian effects on blood flow in large arteries. Computer Methods in Biomechanics and Biomedical Engineering, 4, pp. 149-163, 2001. doi:10.1080/10255840008908002

[17] Shimano, K, Yonezu, S. and Enomoto, Y., Acceleration of Unsteady Incompressible Flow Calculation Using Extrapolation Method, Transactions of Japan Society of Mechanical Engineers, Series B, 74(745), pp. 1896-1902, 2008.

[18] Shimano, K, Yonezu, S. and Enomoto, Y., Acceleration of Unsteady Incompressible Flow Calculation Using Extrapolation Method, Proc. of the fourth International Conference on Computational Fluid Dynamics (ICCFD4), eds Deconinck, H. \& Dick, E., Springer: Berlin, pp. 303-308, 2009.

[19] Wahap, G., Kobori, T., Takakura, Y., Arai, N., Konishi, Y. \& Fukasaku, K., Numerical simulation of Flows in a Pipe with an Aneurismal Sac (Effects of Aneurismal Models and Stents). Advanced Materials Research, 33-37, pp. 1025-1030, 2008. doi:10.4028/www.scientific.net/ AMR.33-37.1025

[20] Alkhamis, T.M., Beissinger, R.L. \& Chediak, J.R., Red blood cell effect on platelet adhesion and aggregation in low-stress shear flow -Myth of Fact? Transactions of American Society of Artificial Internal Organs, 34, pp. 868-873, 1988.

[21] Evans, E.A., Waugh, R. \& Melnik, L., Elastic area compressibility modulus of red blood cell membrane. Biophysical Journal, 16, pp. 585-595, 1976. doi:10.1016/S0006-3495(76)85713-X 